\title{
99
}

\section{Technology tools and the Curie Internet delivery system}

\author{
Gerald W. Meisner \\ Harol M. Hoffman \\ University of North Carolina \\ Greensboro \\ USA
}

\begin{abstract}
We describe CURIE, an information management and delivery system for teachers being developed in the TechTools for Science and Mathematics Learning project. TechTools is a professional development program which emphasizes the use of technological tools in middle and high school science and mathematics classrooms for purposes of enriching the student learning environment and promoting the development of problem solving skills. Teachers receive training in the use of TechTools for data acquisition and analysis, scientific communication and collaboration, and computer modelling and simulation.

The information server CURIE is both a repository of peer reviewed on-line publications in science and mathematics, and a transparent gateway to the Internet.
\end{abstract}

Main conference themes: teacher education

Educational areas: secondary education

Study topics: science/engineering, mathematics

Secondary keywords: collaborative learning, computing, pedagogy, telecommunications, tools 


\section{INTRODUCTION}

This paper describes an Internet delivery system and professional development workshop program for teachers which is being developed at the University of North Carolina, Greensboro with the help of a grant from the National Science Foundation. TechTools for Science and Mathematics Learning (TechTools) is a training program which emphasizes the effective pedagogical use of technological tools in high school science and mathematics classrooms.

The TechTools program is intended to:

- Develop learning environments more suitable for guided inquiry methods of science learning.

- Increase student and teacher access to high quality, hands-on instructional resources in science and mathematics.

- Permit teachers to communicate with each other and with other educators and practising scientists for purposes of sharing instructional materials and classroom techniques, of providing feedback on the TechTools instructional resources database and of reducing feelings of stress and classroom isolation.

- Provide specific examples and build a foundation for learning how to initiate projects in the classroom where students can develop, execute, analyze and publish with the help of information tools, particularly those available on the Internet.

The information server CURIE is a transparent gateway to services on the Internet and also serves as a repository of peer reviewed on-line publications in science and mathematics. It is the primary link to information retrieval tools which will aid in changing the paradigm of classroom teaching from information contained within a single room to that contained within a single building (the library, for example) to that contained on the World Wide Web (WWW) of information. We will examine and evaluate teachers' ability to use technology to enrich the student learning environment in ways which enable and encourage students to define problems of their interest, to seek information necessary for solutions and to develop a vested interest in publishing their findings. We will document the extent to which students benefit from this problem centred learning setting as measured by increases in student achievement in the classrooms of TechTools teachers.

\section{Statement of the problem}

Two problems currently plague the United States' science and mathematics programs. The first concerns quality of instruction, the second has to do with 
demographics. Instruction in the sciences continues to be bound by subject matter categories as opposed to an emphasis on the integration and application of scientific and technological concepts. Laboratory experiences and technological applications in both science and mathematics are insufficient or altogether lacking [1]; according to the US OTA report [2] this is not because schools lack basic hardware, but because they lack:

- innovative instructional software;

- advanced hardware; and

- teacher training in computer use.

Inadequately trained teachers is the most acute deficit. Teachers are illequipped to guide hands-on learning or handle advanced technologies. The OTA report states that although $98 \%$ of US. schools have at least one computer, and although the national ratio of students to computers is $18: 1$, only $50 \%$ of the nation's teachers report ever having used a computer; and the more complex the technology the fewer teachers who feel prepared to use it.

A second exigency confronting science and technology instruction in the US. is that of demographics. By the year $200085 \%$ of people entering the work force will be females, minorities and immigrants. Only $15 \%$ of new workers will be white males. The nation has a responsibility to see that minorities and women who will comprise the bulk of the future work force, are prepared for the jobs of the future. This is particularly true in the fields of science and engineering if a personnel crisis is to be avoided. Yet, women and non-Asian minorities are poorly represented in scientific fields. Women accounted for only $16 \%$ of the Science/Engineering work force in 1988 and just $4 \%$ of the engineers. Blacks accounted for $2.6 \%$ of this work force [3]. In 1989 , women received only $15 \%$ of bachelor's degrees awarded in physics and engineering [3] and experts' predictions for the future remain grim.

The statistics are clear. The progress of minorities and females in mathematics, science and technology will be a major determinant of how close the US. comes to meeting its target goals in these fields. Yet, at the present time US. schools are making it difficult for young women and minorities to pursue careers in science and technology; a problem compounded for minority females who belong to both disadvantaged groups. Negative stereotypes, biased teacher behaviour, social pressures, lack of role models, falsely perceived differences in aptitude and ability combine to form nearly insurmountable barriers to achievement in science and mathematics for minorities and females.

Technology may hold the key to reforming US science and mathematics classrooms. Access to technology and the development of technological skills 
can facilitate the inclusion of minorities and females in the scientific enterprise. Teaching practices which employ technology can be used to level the playing field, to promote achievement, to develop critical thinking skills, to encourage scientific literacy and to facilitate relevant learning experiences in settings which include adequate support systems for students traditionally disadvantaged in the acquisition of scientific training. Thus, a critical juncture exists for implementing serious reform measures in science and mathematics instruction. Professional development programs which are geared toward training teachers in the instructional use and equity potential of technological tools in the classroom, provide the opportunity.

\section{Program description}

\section{Workshop structure}

TechTools inservice professional development workshops are scheduled to be offered annually at 10 university sites across the state of North Carolina. The workshops at each site will serve two teachers from each of 4 schools from each of two school systems. Each year's inservice program will include two five-day workshop sessions in the summer followed by three two-day workshops throughout the school year, interspersed with site visits from project staff. Between workshop sessions teachers will be using the CURIE server to:

- communicate with each other and with project personnel;

- access computer based instructional resources which will be field tested with students in their classrooms;

- develop joint projects among schools;

- participate in building the TechTools databases;

- initiate student centred projects in collaboration with mentored colleagues;

- direct classroom learning which emphasizes the process of problem solving rather than 'drill \& kill' uses of computers.

Inservice teachers participate in the program for two years covering two workshop cycles in order to thoroughly incorporate the necessary technological tools.

\section{Workshop content}

The workshop program emphasizes a systematic approach for teachers to follow in developing experiments for their students on the basis of guided inquiry learning. Teams of two teachers at each workshop will learn how to help their students in designing experiments, developing and writing procedures, obtaining information and data over the Internet, analyzing results by using a 
variety of TechTools, developing computer models and communicating conclusions. This is done both by using multimedia authorware tools and by disseminating information over the CURIE delivery system. The workshops serve to underline the importance of developing basic conceptual lesson modules which place all students as planners, observers, analyzers and publishers of experiments. But also serve to guide teachers through the process of integrating recent TechTools into all phases of their science and mathematics curriculum.

Among the many TechTools to be used in the program are:

Databases to classify events and objects based on observed features or characteristics and to find relationships among objects or events using both similarities and differences;

Spreadsheets to store and process data, to explore their effective representation, to perform mathematical operations and to export data into graphical analysis programs;

Laser discs to access large databases of still pictures and movies, to make measurements of variables and analyze their relationships;

Simulations and computer modelling to conduct experiments which are too dangerous, too expensive, too time consuming or too complex to do in the classroom;

$C D-R O M$ to access large databases of text, numerical data or visuals to use in manners described above;

Probeware to introduce measurements not possible by human senses or with traditional measuring devices found in the classroom;

Internet to expeditiously locate information relevant to the task at hand; digitized video, either taken on site or using 'canned' material - to appeal to students' interest and sense of ownership as well as initiate them into the power of digital processing.

A separate but important matter is the new manner which the teacher can now use to present material in a multimedia format. Teachers will study authoring tutorials, prepare classroom presentations of content material and show them to their colleagues.

Applications in which content specialists will use various TechTools in specific disciplines, include the following:

Biology: Data collection can be automated in a number of different contexts which results in a considerable savings of time, money, and specimens. 
Physiological data on heart-rate in response to various hormones and drugs, muscle contraction in response to varied stimuli, and ventilation rates and volumes under differing circumstances can all be collected at precisely timed intervals.

The use of CD-ROM can also simplify the logistics of simulated presentations of anatomical structure without the increasingly frequent objections of those concerned with the 'rights' of the objects of study.

Chemistry: This area is attractive for the application of TechTools because of

- the expense of chemicals and laboratory equipment to school systems;

- safety factors in handling chemicals;

- the length of time necessary to set up, perform, and clean up a laboratory experiment.

As a substitute for experimentation which is either too expensive or too dangerous TechTools' software and peripherals are available which allow the presentation of experiments based on the use of the video screen to simulate chemical reactivity or instrumentation.

The TechTools program in chemistry includes two major areas. The first uses computer simulation with information databases and video images and action sequences to present individualized chemical investigations for students in the classroom.

The second area of application is the use of inexpensive, computer controlled devices to simulate chemical instrumentation. Results will be used to provide analyses or investigations analogous to those performed in sophisticated laboratories. The excitement and challenge of experimentation in chemistry is learned in a convenient, economical environment.

Physics: Use of TechTools permits the teacher to emphasize the process as well as the function of scientific exploration in a guided manner. High speed data collection probes, interfacing and desk top computers allow real time exploration and analysis of countless physical situations, most of which are suitable in a modified form at the high school and middle school level. A combination of probeware, simulations and image processing techniques are used in workshops to focus the ways in which technology can bring the excitement of the scientific process to the study of motion, electricity and thermal physics. Other activities which are less specific and more integrative in nature will use search engines on the Web to gather data already published.

Mathematics: This discipline will emphasize three uses of TechTools in mathematics pedagogy and concept learning:

- the use of a standard graphing package to understand standard transcendental functions - exponentials, logs, trigonometric functions. The 
emphasis is on trying to develop an appreciation for the global growth properties of functions which show up in physical and economic problems;

- the use of computer graphics to illustrate basic ideas from elementary probability and statistics;

- the use of computer graphics to introduce basic ideas about chaotic dynamics.

\section{CURIE: THE TECHTOOLS DELIVERY SYSTEM.}

The information management and delivery system for reaching the TechTools' goals has been developed by a team of faculty and students at University of North Carolina (UNC), Greensboro. CURIE has capabilities both as an on-line, peer reviewed publishing environment for teachers and a provider of Internet services in a transparent, consistent manner. Understanding and using this communication backbone is woven into the workshop from the first day until the last. Teachers will have client software installed on their platform, either Windows or Macintosh. A SLIP/PPP connection over a $14.4 \mathrm{kbps}$ modem links the teacher to the CURIE server at UNC Greensboro via campuses of the University of North Carolina backbone. Text and graphics material are easily transmitted.

CURIE is the TechTools link to the Internet providing teachers and their students with seamless access in ways which could not otherwise easily be done, to data and graphics, to each other and to the outside world, as well as to the instructors. Confusing (to the uninitiated) protocols for acquiring and using $\mathrm{ftp}$ (a standardized file transfer protocol), telnet (a standardized telecommunications protocol), WWW, for example, are replaced with menu driven options. Student/teacher research teams collaborate on projects, working to solve scientific problems through joint sharing of information and resources. Thus students are given opportunities (as a result of modern technology) to learn to conduct research in the way the scientific enterprise is carried out at the professional level. Access to teleconferencing is available at each institution, using the CU-SeeMe software developed at Cornell University [4]. It allows digitized video and audio to be sent inexpensively over the Internet; an idea which is not new, but previously could only be handled by separate facilities and expensive hardware. To send pictures requires an inexpensive camera and digitizing hardware which comes with newer Macs and can be added to older ones. We also use QuickCam to directly input digitized signals, thus eliminating the need for an expensive digitizing card. 


\section{CURIE is composed of three components:}

\section{Instructional materials database on CURIE}

A major element of this component is the development by the participants themselves of measurement based activities, executed by students and guided by classroom teachers. Learning about directed inquiry activities by using these activities and developing two high-quality, peer reviewed modules by each teacher occurs during the summer workshops. The activities emphasize conceptual development as opposed to, say, verification of principles. Activities which feature data sharing by students across the state are stressed. Field testing occurs by each cohort of teachers during the academic year. The last two of the two-day workshops during the academic year will be devoted to rewriting of the modules, incorporating suggested improvements and uploading the activities into CURIE's data base of similar files. These files can then be accessed by anyone over Internet. This Database Module has a dynamic capability which will diminish teachers' feelings of isolation by allowing them to communicate with each other and also with university scientists and educators regarding field testing of instructional materials, problem solving and other issues of general professional interest. Already housing over 800 labs, demonstrations and similar files this program will result in the addition of 200 files for each of the four years of the program.

All instructional resources included in the databases will be field tested by classroom teachers through the TechTools instructional development program, and will be shown to meet national standards in science and mathematics as well as applying to competencies listed by the state of North Carolina. Use of the database provides opportunities allowing relational links which support general teacher communications and cooperation. At the end of this program CURIE will house 1600 field tested labs, demonstrations and articles by teachers describing what works in the classroom .

\section{Internet search engines and services}

Information search and retrieval on the Web presents a greater hurdle to teachers than does the technology. It is often easier to become lost in 'information space' than it is to find the desired material. We ameliorate this problem by providing a 'layered' approach to information. For each discipline we choose the 20 home pages best suited as educational resources for middle and high school teachers, we list their Universal Resource Locators (a URL has the form http://......./) and provide concise but revealing annotations for each. Behind that list of 20 we provide additional annotated URLs, often of a more specialized type.

Teachers are directed to pointers on the Web as well as to search engines on Gopher and the Web. FTP is supported with common shareware and 
freeware. Tutorials are extensive, with continued feed back from teachers in the field. Emphasis is on the management of information access and retrieval consistent with the vast capabilities of the Internet.

\section{Technical communications support}

This module provides teachers with computer based professional assistance in science and mathematics instruction, and will provide opportunities for development of their professional knowledge and skills. E-mail in the form of questions directed to professional educators, scientists, and business professionals involved with TechTools will permit teachers to receive assistance from experts on matters related to solving classroom problems, implementing specific technological innovations and assessing their science and mathematics curricula. This can be a giant step towards removing the sense of isolation many teachers experience.

\section{RESULTS AND FUTURE DIRECTIONS}

Future directions into which we are heading with CURIE and the development tools include:

- ZooTech: a program for linking appropriate zoos throughout the world with classroom teachers and university mentors;

- collaborative arrangements between elementary and secondary school students with student teachers in different states across the country, in a manner which will be modelled after the Albuquerque Academy in New Mexico;

- inclusion of traditional university courses on the CURIE home page to enable tomorrow's students with different time and economic constraints to access university offerings in more flexible settings. An introductory astronomy course is currently at our site as part of this effort.

At the conference we will present evaluation results from the first phase of TechTools. Implementation of discipline specific workshop modules and the role played by CURIE in the first summer of this four year program will be summarized. 


\section{REFERENCES}

1. Carnegie Commission report on Science, Technology and Government, cited in Why Johnny Can't Do Science and Math. Arthur Fisher, Popular Science, September, 1992, pp. 50-57.

2. Power On! New Tools for Teaching and Learning. (1988). (Report \#OTA-SET-439). Washington, DC: Congress of the United States, Office of Technology Assessment.

3. Women and Minorities in Science and Engineering, National Science Foundation 90-301, January 1990.

4. CU-SeeMe was adopted by the National Science Foundation for its Global Schoolhouse project which links schoolrooms to the Internet. 